\title{
Ultrasonography for the Diagnosis of Pneumothorax after Transbronchial Lung Cryobiopsy in Diffuse Parenchymal Lung Diseases
}

\author{
Luca Viglietta $^{a}$ Riccardo Inchingolod Cristina Pavano ${ }^{\mathrm{e}}$ Sara Tomassetti ${ }^{\mathrm{a}}$ \\ Sara Piciucchi ${ }^{b}$ Andrea Smargiassid ${ }^{d}$ Claudia Ravaglia ${ }^{a} \quad$ Alessandra Dubinic $^{c}$ \\ Christian Guriolia Carlo Gurioli ${ }^{a}$ Venerino Poletti ${ }^{a}{ }^{f}$ \\ Departments of aThoracic Diseases, ${ }^{\mathrm{b}}$ Radiology, and ${ }^{\mathrm{C} P a t h o l o g y, ~ G . B . ~ M o r g a g n i-L . ~ P i e r a n t o n i ~ H o s p i t a l, ~ F o r l i ̀, ~}$ \\ dPulmonary Medicine Department, Fondazione Policlinico Universitario A. Gemelli, Rome, and e Pulmonology Unit, \\ Policlinico G. Rodolico, University of Catania, Catania, Italy; ' Department of Respiratory Diseases and Allergy, \\ Aarhus University Hospital, Aarhus, Denmark
}

\section{Keywords}

Interstitial lung disease $\cdot$ Pneumothorax · Radiology and other imaging $\cdot$ Bronchoscopy and interventional techniques

\begin{abstract}
Background: Transbronchial lung cryobiopsy (TBLC) can be indicated in diffuse parenchymal lung diseases (DPLDs) when a confident noninvasive diagnosis cannot be made. The 2 most relevant complications of TBLC are bleeding and pneumothorax (PTX). The accuracy of chest ultrasonography (US) for the detection of PTX is higher when compared to chest X-ray (CXR) with reference to computed tomography (CT) scan as a gold standard. Objective: We evaluated the accuracy of chest US in detecting PTX after TBLC in patients with DPLDs. Methods: Patients underwent TBLC during rigid bronchoscopy in deep sedation. Cryobiopsy was performed with fluoroscopic guidance. Three hours later, patients underwent chest US and standard CXR. When there was no concordance between chest US and CXR, chest CT was required. Results: Forty-three patients were enrolled into the study. Cryobiopsy was performed in the right lung in 36
\end{abstract}

\section{KARGER}

(C) 2017 S. Karger AG, Basel

E-Mail karger@karger.com

www.karger.com/res
(84\%) patients. PTX was diagnosed in 10 (23\%) patients by CXR. There was complete agreement between radiologists interpreting CXR $(k=1,95 \% \mathrm{Cl} 1)$. Chest US was positive for PTX in 11 (25\%) patients. There was complete agreement between pulmonologists interpreting chest US $(k=1,95 \% \mathrm{Cl}$ 1). The prevalence of PTX diagnosed by concordance of CXR and chest US was 23\% (10/43, 95\% Cl 11.8-38.7). The sensitivity and specificity of chest US were $90 \%$ (95\% CI 55.5-99.7) and $94 \%$ (95\% Cl 79.8-99.3), respectively. Moreover, the positive and negative predictive values were $82 \%$ (95\% Cl $48-$ 98) and $97 \%$ (95\% Cl 84-100), respectively. Conclusion: Chest US is a highly sensitive and specific diagnostic tool for the diagnosis of PTX after TBLC.

(c) 2017 S. Karger AG, Basel

\section{Introduction}

According to American Thoracic Society/European Respiratory Society international guidelines, surgical lung biopsy (SLB) is indicated in diffuse parenchymal lung diseases (DPLDs) when a confident noninvasive diagnosis cannot be made. However, SLB has appreciable

Luca Viglietta, MD

U.O. Pneumologia

Via Carlo Forlanini, 34

IT-47121 Forli (Italy)

E-Mail viglietta.luca@gmail.com 
costs and risks (including a mortality of $1.7 \%$ within 90 days for elective procedures) [1]. The majority of subjects are excluded because of advanced age, comorbidities, severe respiratory impairment, and pulmonary hypertension, and a considerable minority refuse to undergo surgery. Transbronchial lung cryobiopsy (TBLC) has clearly demonstrated a superior diagnostic yield in comparison to conventional forceps biopsy (80 vs. $30 \%$ ) [2, 3], close to that of SLB (98\%) [4]. Mortality of TBLC is significantly lower than that of SLB (0.1 vs. 1.7\%) [3]. All other complications (fever, transient respiratory failure, and empyema), hospitalization lengths, and costs are significantly lower for TBLC than for SLB. For all these reasons, TBLC is significantly changing the diagnostic algorithm of DPLDs and in many expert centers has already replaced SLB in the vast majority of cases.

When dealing with TBLC, the 2 most relevant complications that need to be timely and properly managed are bleeding and pneumothorax (PTX). PTX is very rare during bronchoscopy with transbronchial biopsy (210\%) [5]. The prevalence of PTX after cryobiopsy varies from 4.9 to $20.2 \%$ in patients with DPLD [3, 6-8]. Some studies have shown that development of PTX occurs within the first hour after the procedure, even though it might be invisible on chest X-rays (CXRs) performed immediately after bronchoscopy; there is no evidence of the occurrence of PTX even after $4 \mathrm{~h}$ by transbronchial biopsy [9].

CXR used routinely for PTX diagnosis has a sensitivity of $46 \%$ [10]; the classic posterior-anterior projection in more difficult cases may be associated with a lateral view, and expiratory X-ray is not necessary [11]. Chest computed tomography (CT) represents the gold standard for the diagnosis of PTX. In recent years, use of chest ultrasonography (US) has spread for the diagnosis of PTX; it reaches a pooled sensitivity of $87 \%$ (95\% CI 81-92\%) and a specificity of 99\% (95\% CI 98-99) [12] according to a standardized method searching for specific pathognomonic signs [13].

Chest US has more diagnostic power than CXR with reference to CT scan as a gold standard. Moreover, accuracy of US for the detection of PTX is higher than that of CXR with reference to CT scan as a gold standard. However, although CT is more sensitive than CXR, it is performed only in uncertain cases to avoid excess of radiation in often young patients [1].

Chest US in PTX diagnosis after TBLC has never been evaluated, but its use in place of traditional CXR would have some advantages, avoiding exposure to radiation and reducing costs of health care and hospital stay. This

Ultrasound for Pneumothorax after

Cryobiopsy in DPLDs study aims to evaluate the accuracy of chest US in detecting PTX after TBLC in patients with DPLD. Secondary aims of this study are to assess postprocedure PTX prevalence and concordance between operators for both CXR and chest US in detecting PTX.

\section{Materials and Methods}

We enrolled patients with DPLD who underwent TBLC for a definite diagnosis from December 2015 to February 2016 at the Interventional Pulmonology Unit, Morgagni Hospital in Forli, Italy. Patients had to be at least 18 years old, with a forced vital capacity $>50 \%$ of the predicted normal value. Patients with coagulopathy (platelet count $<70,000 \times 10^{9} / \mathrm{L}$, prothrombin time international normalized ratio $>1.5$ ), diffuse bullous disease, hemodynamic instability, and severe hypoxemia $\left(\mathrm{PaO}_{2}<50 \mathrm{~mm} \mathrm{Hg}\right.$ on room air) as well as pregnant patients were excluded.

The following clinical and functional data were collected: age, cigarette smoking and pack years, weight, height, body mass index, oxygen saturation on room air, vital capacity, forced vital capacity, forced expiratory volume in $1 \mathrm{~s}$, diffusing lung capacity for carbon monoxide, functional residual capacity, total lung capacity, and residual volume. Radiological data collected in the CT scan performed in the last 2 months were lobular location of honey combing, subpleural cysts, and emphysema.

Patients underwent TBLC during rigid bronchoscopy in deep sedation with assistance of an anesthesiologist as previously published [14]. Cryobiopsy was performed with fluoroscopic guidance that can also immediately rule out postprocedure massive PTX. Fluoroscopic imaging was interpreted by the interventional pulmonologist.

Three hours after the procedure, patients underwent the following examinations:

- Arterial oxygen saturation $\left(\mathrm{SaO}_{2}\right)$ evaluation.

- Chest US performed by 2 resident pulmonologists who attended a 3-day theoretical and practical chest US course and performed 30 chest US to achieve competency before starting this study [15]. Pulmonologists were blinded to the site of cryobiopsy and colleagues' chest US results. Sonoscape SSI-600 with a 5- to $10-\mathrm{MHz}$ linear probe was used in patients in supine position. US images were studied in a longitudinal and transversal scanning plane. Examinations were carried out from the clavicle to the diaphragm at the intercostal spaces on both hemithoraxes in parasternal, midclavicular, anterior axillary, and midaxillary lines in B-mode and $\mathrm{M}$-mode scans. The combination of 4 ultrasonographic signs allowed the pulmonologist to identify PTX. These signs were: (1) presence of lung point(s), (2) absence of lung sliding, (3) absence of B-lines, (4) absence of lung pulse, and (5) "stratosphere sign" [16].

- Standard CXR in posterior-anterior projection with the patient in the orthostatic position in exhalation and inhalation. CXR was interpreted by the radiologist on duty and reviewed by a second blinded radiologist. Radiologists were blinded also to chest US results.

If PTX was clinically suspected before the time by the attending physician, these exams were preponed. When there was no concordance between chest US and CXR, chest CT was required.
Respiration 2017;94:232-236 DOI: $10.1159 / 000477818$ 
Table 1. Clinical and functional data

\begin{tabular}{ll}
\hline Patients' clinical features & \\
\hline Total, $n$ & 43 \\
Age, years & $63 \pm 12$ \\
Male/female, $n$ & $26 / 17$ \\
$\mathrm{BMI}$ & $27 \pm 4$ \\
$\mathrm{VC}, \%$ & $86 \pm 17$ \\
$\mathrm{FVC}, \%$ & $83 \pm 18$ \\
$\mathrm{FEV}, \%$ & $87 \pm 18$ \\
$\mathrm{TLC}, \%$ & $76 \pm 16$ \\
$\mathrm{FRC}, \%$ & $76 \pm 26$ \\
$\mathrm{RV}, \%$ & $69 \pm 42$ \\
$\mathrm{DLCO}, \%$ & $55 \pm 19$ \\
PaO,$\%$ (before biopsy) & $96 \pm 3$ \\
Smokers, $n$ & 24 \\
Pack/years & $33 \pm 22$ \\
Samples for patient & $3.5 \pm 1.1$ \\
\hline
\end{tabular}

Values are means \pm standard deviations unless otherwise indicated. BMI, body mass index (in $\mathrm{kg} / \mathrm{m}^{2}$ ); $\mathrm{VC}$, vital capacity; $\mathrm{FVC}$, forced vital capacity; $\mathrm{FEV}_{1}$, forced expiratory volume in $1 \mathrm{~s}$; TLC, total lung capacity; FRC, functional residual capacity; RV, residual volume; DLCO, diffusing lung capacity for carbon monoxide; $\mathrm{PaO}_{2}$, arterial pressure of oxygen.

\section{Statistical Analysis}

The population is described with descriptive statistics. Continuous variables are expressed as medians and standard deviations, while categorical variables are expressed as frequency and percentage. Cohen $\kappa$ statistics were calculated to evaluate the interobserver agreement of investigators.

Sensitivity, specificity, positive and negative predictive value, as well as disease prevalence are expressed as percentages. Their confidence intervals are exact Clopper-Pearson confidence intervals. Receiver operating characteristic curve was also obtained. The significance level was set at $p<0.05$.

\section{Statement of Ethics}

The study protocol was approved by the local ethical committee (CE IRST IRCCS - AVR, protocol No. 2015/0289377/P). Informed consent was obtained from all subjects enrolled.

\section{Results}

Forty-four patients were screened, and 43 were enrolled into the study. One patient was excluded because of a final diagnosis of lung cancer. Clinical and functional data of patients are described in Table 1.

Cryobiopsy was performed in the right lung in 36 (84\%) patients and in the left lung in 7 (16\%). PTX was diagnosed in $10(23 \%)$ patients by CXR. There was complete agree-
Table 2. US results for the diagnosis of PTX

\begin{tabular}{lrlr}
\hline PTX yes $(n=10)$ & \multicolumn{3}{l}{ PTX no $(n=33)$} \\
\hline US correct positive & 9 & US false positive & 2 \\
US false negative & 1 & US correct negative & 31 \\
\hline Total & 10 & Total & 33 \\
\hline
\end{tabular}

US, ultrasound; PTX, pneumothorax.

Table 3. Chest US diagnostic performance in the detection of PTX

\begin{tabular}{lcc}
\hline Index & Value & $95 \%$ CI \\
\hline Sensitivity, \% & 90 & $55.5-99.7$ \\
Specificity, \% & 94 & $79.8-99.3$ \\
Positive likelihood ratio & 14.8 & $3.8-57.8$ \\
Negative likelihood ratio & 0.1 & $0-0.6$ \\
Positive predictive value, \% & 82 & $48-98$ \\
Negative predictive value, \% & 97 & $84-100$ \\
\hline
\end{tabular}

US, ultrasound; PTX, pneumothorax; CI confidence interval.

ment between radiologists interpreting CXR $(k=1,95 \% \mathrm{CI}$ $1)$. All PTX occurred in the right hemithorax. A chest tube was inserted in 4 subjects ( $40 \%$ of the PTX group).

Chest US was positive for PTX in 11 (25\%) patients. There was complete agreement between pulmonologists interpreting chest US ( $k=1,95 \%$ CI 1$)$. All cases of PTX detected in B-mode and defined by the absence of both lung sliding and B-lines were characterized also by "stratosphere sign" that replaced "seashore sign" in Mmode [17].

All PTX requiring drainage were detected by chest US. A massive PTX was diagnosed with fluoroscopy and clinical features in 1 patient. Immediately, chest US was performed (positive for PTX), and a chest tube was inserted.

In 4 (9\%) patients, there was not concordance between CXR and chest US for the detection of PTX, but the patients refused to perform a chest CT because clinical and functional parameters were normal and they preferred to avoid radiation. One patient (2\%) was negative on chest US and positive on CXR only in the expiratory scan (PTX $4 \mathrm{~mm}$ ). Three patients (6\%) were positive on chest US (presence of lung point) and negative on CXR. One of these developed acute dyspnea $5 \mathrm{~h}$ after cryobiopsy. The patient underwent a second CXR that detected PTX, and a chest tube was inserted. 
Table 4. Final diagnosis of interstitial lung disease from multidisciplinary discussion

\begin{tabular}{llll}
\hline Final multidisciplinary diagnosis & $n$ & $\%$ & Rate of PTX \\
\hline IPF & 18 & 42 & $7(70 \%)$ \\
OP & 4 & 9 & $1(10 \%)$ \\
SRIF & 4 & 9 & 0 \\
HP & 3 & 7 & $1(10 \%)$ \\
NSIP & 2 & 5 & $1(10 \%)$ \\
Lymphoma & 2 & 5 & 0 \\
Bronchioliotis & 2 & 5 & 0 \\
Sarcoidosis & 2 & 5 & 0 \\
CPFE & 1 & 2 & 0 \\
IgG4-RD & 1 & 2 & 0 \\
IPAF & 1 & 2 & 0 \\
PVOD & 1 & 2 & 0 \\
Eosinophilic pneumonia & 1 & 2 & 0 \\
Nondiagnostic & 1 & 2 & 0 \\
\hline
\end{tabular}

PTX, pneumothorax; IPF, idiopathic pulmonary fibrosis; OP, organizing pneumonia; SRIF, smoking-related interstitial fibrosis; HP, hypersensitivity pneumonitis; NSIP, nonspecific interstitial pneumonia; CPFE, combined pulmonary fibrosis and emphysema syndrome; IgG4-RD, immunoglobulin G4-related disease; IPAF, interstitial pneumonia with autoimmune features; PVOD, pulmonary veno-occlusive disease.

The prevalence of PTX diagnosed by concordance of CXR and chest US was 23\% (10/43, 95\% CI 11.8-38.7). The diagnostic performance of chest US in PTX detection is reported in Tables 2 and 3.

The analysis showed that the sensitivity and specificity of chest US were 90\% (95\% CI 55.5-99.7) and 94\% (95\% CI 79.8-99.3), respectively. Moreover, the positive and negative predictive values were $82 \%$ (95\% CI $48-98)$ and 97\% (95\% CI 84-100), respectively. The area under the receiver operating characteristic curve for chest US was 0.92 (95\% CI 0.79-0.98; $Z$ test: $p<0.0001)$.

The final multidisciplinary interstitial lung disease diagnosis is reported in Table 4. Idiopathic pulmonary fibrosis was diagnosed in $42 \%$ of patients, and $39 \%$ of them developed PTX after TBLC.

\section{Discussion}

In this prospective pilot study, we showed that chest US is feasible and accurate for the detection of PTX after TBLC in patients with DPLD. To our knowledge, this is the first experience reported in the literature. In particular, the rapid bedside detection of the absence of both slid- ing sign and B-lines in patients who usually showed diffuse and bilateral vertical artifacts [17-20] could optimize the postprocedural management of patients with DPLD who underwent TBLC.

The larger sample of the lung parenchyma resulting from a TBLC is expected to increase the risk of PTX significantly as compared to flexible forceps biopsy. The prevalence of PTX varies from 4.9 to $20.2 \%$ [3, 6-8]. The rate of PTX after TBLC is higher than the usually reported rate observed with the flexible forceps biopsy [21-26]. Our group described a prevalence of PTX of $23 \%$, and the incidence of PTX was independent of the number and size of the biopsy sample $[27,28]$.

In our study, PTX occurred in one-fourth of cases. The higher incidence of PTX, compared to previously mentioned ones $[3,6-8]$, may be partly explained by a high percentage of patients who suffered from idiopathic pulmonary fibrosis enrolled in the study (42\%).

The first experience with the accuracy of chest US in the diagnosis of postinterventional PTX and a comparison to CXR in patients who underwent transbronchial biopsy were reported by Reissig and Kroegel [29] in 2005. Subsequently, Kreuter et al. [30] evaluated the significance of US of the chest in the diagnosis of PTX supplemented by power color Doppler imaging. The authors found high values of sensitivity, specificity, and accuracy of chest US with reference to CXR as a gold standard. A more recent report by Kumar et al. [23] prospectively evaluated the accuracy of chest US in the detection of PTX after transbronchial lung biopsy. The authors demonstrated a good sensitivity of US in detecting PTX following transbronchial lung biopsy. A limitation of this study was a lack of comparative chest CT when there was no concordance between US and CXR; however, in 3 patients positive on US and negative on CXR, the presence of lung points allowed PTX diagnosis with a high degree of confidence.

Our findings of diagnostic accuracy of chest US for the diagnosis of postprocedural PTX corroborate the results of prior studies. A novel aspect of this study is that we extended the advantages of US to patients suffering from DPLD and undergoing TBLC for a definitive diagnosis. Particularly, our approach exploits the ready availability of US that allows the pulmonologist who performed the bronchoscopy to detect postprocedural PTX within a short time and optimizes the use of ionizing radiation. The main conclusion of our work is that chest US is a highly sensitive and specific diagnostic tool for the diagnosis of PTX after TBLC. 


\section{References}

$\checkmark 1$ Hutchinson JP, Fogarty AW, McKeever TM, Hubbard RB: In-hospital mortality after surgical lung biopsy for interstitial lung disease in the United States. 2000 to 2011. Am J Respir Crit Care Med 2016;193:1161-1167.

-2 Tomassetti S, Cavazza A, Colby TV, Ryu JH, Nanni O, Scarpi E, Tantalocco P, Buccioli M, Dubini A, Piciucchi S, Ravaglia C, Gurioli C, Casoni GL, Gurioli C, Romagnoli M, Poletti $\mathrm{V}$ : Transbronchial biopsy is useful in predicting UIP pattern. Respir Res 2012;13:96.

>3 Ravaglia C, Bonifazi M, Wells AU, Tomassetti S, Gurioli C, Piciucchi S, Dubini A, Tantalocco P, Sanna S, Negri E, Tramacere I, Ventura VA, Cavazza A, Rossi A, Chilosi M, La Vecchia C, Gasparini S, Poletti V: Safety and diagnostic yield of transbronchial lung cryobiopsy in diffuse parenchymal lung diseases: a comparative study versus video-assisted thoracoscopic lung biopsy and a systematic review of the literature. Respiration 2016;91: 215-227.

-4 Tomassetti S, Wells AU, Costabel U, Cavazza A, Colby TV, Rossi G, Sverzellati N, Carloni A, Carretta E, Buccioli M, Tantalocco P, Ravaglia C, Gurioli C, Dubini A, Piciucchi S, Ryu $\mathrm{JH}$, Poletti V: Bronchoscopic lung cryobiopsy increases diagnostic confidence in the multidisciplinary diagnosis of idiopathic pulmonary fibrosis. Am J Respir Crit Care Med 2016;193:745-752.

5 Patel RR, Utz JP: Bronchoscopic lung biopsy; in Wang K-P, Metha AC, Turner JF Jr (eds): Flexible Bronchoscopy. Chichester, WileyBlackwell, 2011, pp 117-131.

6 Dhooria S, Sehgal IS, Aggarwal AN, Behera D, Agarwal R: Diagnostic yield and safety of cryoprobe transbronchial lung biopsy in diffuse parenchymal lung diseases: systematic review and meta-analysis. Respir Care 2016; 61:700-712.

$\checkmark 7$ Johannson KA, Marcoux VS, Ronksley PE, Ryerson CJ: Diagnostic yield and complications of transbronchial lung cryobiopsy for interstitial lung disease: a systematic review and meta-analysis. Ann Am Thorac Soc 2016; 13:1828-1838.

$>8$ Gershman E, Fruchter O, Benjamin F, Nader AR, Rosengarten D, Rusanov V, Fridel L, Kramer MR: Safety of cryo-transbronchial biopsy in diffuse lung diseases: analysis of three hundred cases. Respiration 2015;90:40-46.

-9 Boskovic T, Stojanovic M, Stanic J: Pneumothorax after transbronchial needle biopsy. J Thorac Dis 2014;6:S427-S434.
0 Ball CG, Kirkpatrick AW, Laupland KB, Fox DL, Litvinchuk S, Dyer DM, Anderson IB, Hameed SM, Kortbeek JB, Mulloy R: Factors related to the failure of radiographic recognition of occult posttraumatic pneumothoraces. Am J Surg 2005; 189:541.

11 Tschopp JM, Bintcliffe O, Astoul P, et al: ERS task force statement: diagnosis and treatment of primary spontaneous pneumothorax. Eur Respir J 2015;46:321-335.

-12 Ebrahimi A, Yousefifard M, Mohammad Kazemi H, Rasouli HR, Asady H, Moghadas Jafari A, Hosseini M: Diagnostic accuracy of chest ultrasonography versus chest radiography for identification of pneumothorax: a systematic review and meta-analysis. Tanaffos 2014;13:29-40.

13 Husain LF, Hagopian L, Wayman D, Baker WE, Carmody KA: Sonographic diagnosis of pneumothorax. J Emerg Trauma Shock 2012; 5:76-81.

14 Poletti V, Hetzel J: Transbronchial cryobiopsy in diffuse parenchymal lung disease: need for procedural standardization. Respiration 2015;90:275-278.

15 Wilkerson RG, Stone MB: Sensitivity of bedside ultrasound and supine anteroposterior chest radiographs for the identification of pneumothorax after blunt trauma. Acad Emerg Med 2010;17:11-17.

16 Lichtenstein DA, Mezière G, Lascols N, Biderman P, Courret JP, Gepner A, Goldstein I, Tenoudji-Cohen M: Ultrasound diagnosis of occult pneumothorax. Crit Care Med 2005; 33:1231-1238.

17 Chen L, Zhang Z: Bedside ultrasonography for diagnosis of pneumothorax. Quant Imaging Med Surg 2015;5:618-623.

18 Hasan AA, Makhlouf HA: B-lines: Transthoracic chest ultrasound signs useful in assessment of interstitial lung diseases. Ann Thorac Med 2014;9:99-103.

19 Reissig A, Copetti R: Lung ultrasound in community-acquired pneumonia and in interstitial lung diseases. Respiration 2014;87:179189.

20 Vizioli L, Ciccarese F, Forti P, Chiesa AM, Giovagnoli M, Mughetti M, Zompatori M, Zoli M: Integrated use of lung ultrasound and chest X-ray in the detection of interstitial lung disease. Respiration 2017;93:15-22.
1 Du Rand IA, Blaikley J, Booton R, Chaudhuri N, Gupta V, Khalid S, Mandal S, Martin J, Mills J, Navani N, Rahman NM, Wrightson JM, Munavvar M; British Thoracic Society Bronchoscopy Guideline Group: British Thoracic Society guideline for diagnostic flexible bronchoscopy in adults: accredited by NICE. Thorax 2013;68(suppl 1):i1-i44.

22 Izbicki G, Shitrit D, Yarmolovsky A, Bendayan D, Miller G, Fink G, Mazar A, Kramer MR: Is routine chest radiography after transbronchial biopsy necessary?: A prospective study of 350 cases. Chest 2006;129:1561-1564.

23 Kumar S, Agarwal R, Aggarwal AN, Gupta D, Jindal SK: Role of ultrasonography in the diagnosis and management of pneumothorax following transbronchial lung biopsy. J Bronchology Interv Pulmonol 2015;22:14-19.

24 Ibrahim AS, Allangawi MH, Sattar HA, Mobyed HS, Almohammed AA: Indications, diagnostic yields and complications of transbronchial biopsy over 5 years in the State of Qatar. Saudi Med J 2005;26:641-645.

25 Hue SH: Complications in transbronchial lung biopsy. Korean J Intern Med 1987;2: 209-213.

26 Izbicki G, Romem A, Arish N, Cahan C, Azulai H, Chen-Shuali C, Tennenhaus E, Bar-Yosef Z, Zlotkevich E, Rokach A: Avoiding routine chest radiography after transbronchial biopsy is safe. Respiration 2016;92:176-181.

27 Casoni GL, Tomassetti S, Cavazza A, Colby TV, Dubini A, Ryu JH, Carretta E, Tantalocco P, Piciucchi S, Ravaglia C, Gurioli C, Romagnoli M, Gurioli C, Chilosi M, Poletti V: Transbronchial lung cryobiopsy in the diagnosis of fibrotic interstitial lung diseases. PLoS One 2014;9:e86716.

28 Poletti V, Casoni G L, Gurioli C, Ryu JH, Tomassetti S, Lung cryobiopsies: A paradigm shift in diagnostic bronchoscopy? Respirology 2014;19:645-654.

29 Reissig A, Kroegel C: Accuracy of transthoracic sonography in excluding post-interventional pneumothorax and hydropneumothorax. Comparison to chest radiography. Eur J Radiol 2005;53:463-470.

30 Kreuter M, Eberhardt R, Wenz H, Schmitteckert H, Heussel CP, Herth F: Diagnostic value of transthoracic ultrasound compared to chest radiography in the detection of a post-interventional pneumothorax. Ultraschall Med 2011;32(suppl 2):E20-E23. 